TRANSACTIONS OF THE

AMERICAN MATHEMATICAL SOCIETY

Volume 176, February 1973

\title{
FREE VECTOR LATTICES
}

BY

\author{
ROGER D. BLEIER
}

ABSTRACT. An investigation into the algebraic properties of free objects in the category of vector lattices is carried out. It is shown that each ideal of a free vector lattice is a cardinal (direct) sum of indecomposable ideals, and that there are no nonzero proper characteristic ideals.

Questions concerning injective and surjective endomorphisms are answered. Moreover, for finitely generated free vector lattices it is shown that the maximal ideals are precisely those which are both prime and principal.

These results are preceded by an efficient review of the known properties of free vector lattices. The applicability of the theory to abelian lattice-ordered groups is discussed in a brief appendix.

1. Introduction. There are by now a number of articles in the literature on free vector lattices or closely related subjects. Most of these contain a proof of the fundamental theorem that each free vector lattice is a vector lattice of realvalued functions (with pointwise operations). In addition, there are the important algebraic properties discovered by E. Weinberg [13] and [14]. The setting provided by K. Baker [2] seems particularly attractive. One purpose of this paper is to show that the existent theory on free vector lattices can be efficiently developed around the representation theorem $\left({ }^{1}\right)$ in [2]. Beyond that, many new results are obtained.

The paper is divided into two parts and an appendix. Part I is devoted to the general theory of the free vector lattice. The aforementioned review is carried out, and two new results are proved: namely, that free vector lattices are characteristically simple, and that each of their ideals is a cardinal sum of indecomposable ideals.

Part II is concerned only with finitely generated vector lattices. $\$ 3$ provides necessary preliminaries. Let us denote the free vector lattice on $n$ elements ( $n$ finite) by FVLn. A result of $\$ 4$ is that each onto endomorphism of FVLn is oneto-one, while a consequence of the discussion in $\$ 5$ is that there are one-to-one endomorphisms of FVL $n$ that are not onto. In $\$ 6$ it is proved that each maximal

Received by the editors June 1,1971 .

AMS (MOS) subject classifications (1970). Primary 06A65, 06A60.

Key words and phrases. Vector lattice, lattice-ordered group, free, archimedean, finitely generated, representation by continuous functions, maximal ideals.

(1) A similar representation appeared earlier in [11]. 
ideal of a finitely generated vector lattice is a principal ideal. A consequence is that a finitely generated vector lattice admitting only archimedean epimorphic images is necessarily the cardinal sum of a finite number of copies of the real numbers.

The appendix briefly summarizes the applicability of the preceding theory to abelian lattice-ordered groups (l-groups).

The reader is referred to [6], [8], or [10] for the basic algebraic theory of vector lattices and $l$-groups.

The letter $R$ will denote the real numbers. By "vector lattice" we always mean "real vector lattice"; that is, the totally-ordered field of scalars is understood to be $R$. In place of "vector lattice homomorphism" we shall ordinarily write simply "homomorphism". This convention applies, in particular, to epimorphisms, isomorphisms, endomorphisms, and automorphisms.

The subset $S$ of a vector lattice $V$ generates $V$ if no proper vector sublattice of $V$ contains $S$. If $S$ generates $V$, then $V=\left\{\bigvee_{I} \wedge_{J} a_{i j} \mid I, J\right.$ are finite, and each $a_{i j}$ is a linear combination of elements of $\left.S\right\}$. If some finite subset of $V$ generates $V$, then $V$ is finitely generated. We emphasize that this does not, in general, imply that $V$ has finite dimension as a vector space.

A free vector lattice is a vector lattice $F$ with a generating subset $S$ such that if $\alpha$ is a function from $S$ into a vector lattice $V$ then $\alpha$ extends to a homomorphism of $F$ into $V . S$ is then called a free set of generators for $F$. We also say that $F$ is free on $S$. If $S$ is any nonempty set, then, since the class of vector lattices is equationally definable, there exists a unique (up to isomorphism) vector lattice $F$ which is free on $S$. (See [6, pp. 143-144].)

We introduce some further notation and note a few elementary, well-known facts. $V(X)$ will denote the vector lattice of all real-valued functions on the set $X$ with pointwise operations. In other words, $V(X)$ is just the cardinal product of copies of $R$ indexed by $X$.

$T(f)$ will denote the nonzero support of $f \in V(X)$; that is, $T(f)=\{x \in X \mid$ $f(x) \neq 0\}$. Clearly, $T(f)=T(|f|)$, and each of $T(f+g), T(f \vee g)$, and $T(f \wedge g)$ is contained in $T(f) \cup T(g)$ if $f, g \in V(X)$; moreover, if $f, g \geq 0$, then $T(f+g)=$ $T(f) \cup T(g)=T(f \vee g)$ and $T(f \wedge g)=T(f) \cap T(g)$. If $\alpha$ is any bijection of $X$ to itself, then $T\left(f \circ \alpha^{-1}\right)=\alpha(T(f))$.

If $A$ is a nonempty subset of $V(X)$, we let $T(A)=\bigcup\{T(f) \mid f \in A\}$. If $V$ is a vector sublattice of $V(X)$, and $A$ and $B$ are ideals of $V$, then $T(A \vee B)=$ $T(A) \cup T(B)$ and $T(A \cap B)=T(A) \cap T(B)$. Again, these facts are both well known and easily proved.

\section{PART I}

2. The general theory of the free vector lattice. We begin this section by giving necessary and sufficient conditions for a generating subset $S$ of a vector lattice 
$V$ to be a free set of generators. These are then used to prove the main theorem. Weinberg's important results follow as corollaries. Finally, two new theorems about free vector lattices are proved.

Lemma 2.1. If $V$ is a vector lattice, and $x_{1}, \cdots, x_{k} \in V$ are such that $r_{1} x_{1}+\cdots+r_{k} x_{k}=0$ for some real numbers $r_{1}, \cdots, r_{k} \geq 0$ not all 0 , then $\bigwedge_{j=1}^{k} x_{j} \leq 0$.

Proof. The general case follows from the case of $V$ totally ordered since every vector lattice is a subdirect sum of totally ordered vector spaces. But if $V$ is totally ordered and the hypothesis is satisfied, then at least one of the $x_{j}$ must be $\leq 0$, whence $\wedge x_{j} \leq 0$.

Theorem 2.2. Let $S$ be a generating subset of the vector lattice $V$, and let $M$ be the subspace of $V$ generated by $S . V$ is free on $S$ if and only if

(i) $S$ is a vector space bas is for $M$, and

(ii) if $x_{1}, \cdots, x_{k} \in M$ and $\bigwedge_{j=1}^{k} x_{j} \leq 0$, then there exist real numbers $r_{1}$, $\cdots, r_{k} \geq 0$ and not all 0 sucb $r_{1} x_{1}+\cdots+r_{k} x_{k}=0$.

Remark. The necessity of these conditions was noted in [4].

Proof of sufficiency. Since the class of vector lattices is equationally definable, there exists a vector lattice $F$ which is free on $S$. The identity on $S$ extends to a vector lattice epimorphism $\eta: F \rightarrow V$. We need only show $\operatorname{Ker} \eta=0$. Note that the restriction of $\eta$ to the subspace $L$ of $F$ generated by $S$ is a vector space isomorphism by condition (i).

Suppose $x \in F$ and $\eta x=0$. Write $x=\bigvee_{I} \wedge_{J} x_{i j}$ where $I, J$ are finite and the $x_{i j}$ are in $L$. Then $\bigvee_{I} \bigwedge_{J} \eta x_{i j}=0$, and hence $\bigwedge_{J} \eta x_{i j} \leq 0$ for each $i \in I$. Fix $i \in I$. By condition (ii) there exist real numbers $r_{i j} \geq 0$ and not all 0 such that $\Sigma_{J} r_{i j} \eta\left(x_{i j}\right)=0$. Thus $\eta\left(\Sigma_{J} r_{i j} x_{i j}\right)=0$, and since the restriction of $\eta$ to $L$ is an isomorphism, we conclude $\Sigma_{j} r_{i j} x_{i j}=0$. Thus $\bigwedge_{J} x_{i j} \leq 0$ by Lemma 2.1 . Hence $x=\bigvee_{I} \wedge_{J} x_{i j} \leq 0$. Also, $\eta(-x)=0$ and so $-x \leq 0$. Thus $x=0$, and $\eta$ is an isomorphism.

We turn now to the representation theorem in [2]. Our proof features the application of an important theorem from convex geometry: Let $A$ be a finite subset of $R^{n}$ ( $n$ finite), and let $C$ be the convex hull of $A$ in $R^{n}$. Then $C$ can be viewed either as the set of all convex combinations of the elements of $A$ or, alternatively, as the intersection of those closed half-spaces of $R^{n}$ which contain $A$.

Theorem 2.3. Let $S$ be a set. For each $i \in S$, let $\pi_{i}: R^{S} \rightarrow R$ be the ith coordinate projection. The vector sublattice of $V\left(R^{S}\right)$ generated by $\vec{S}=\left\{\pi_{i} \mid i \in S\right\}$ is free with $\bar{S}$ as a free set of generators.

Proof. Condition (i) of The orem 2.2 is satisfied since the $\pi_{i}$ are linearly independent. 
Let $M$ be the subspace of $V\left(R^{S}\right)$ generated by $\bar{S}$, and suppose $g_{1}, \cdots, g_{k} \in M$ are such that $\bigwedge_{j=1}^{k} g_{j} \leq 0$. We wish to show there exist $0 \leq r_{j} \in R$ not all 0 such that $r_{1} g_{1}+\cdots+r_{k} g_{k}=0$. The function values for $g_{1}, \ldots, g_{k}$ depend only on those finitely many $\pi_{i}$ that are needed to express $g_{1}, \ldots, g_{k}$ as linear combinations of the $\pi_{i}$. It follows that without loss of generality we can assume $|S|=n$ is finite.

Thus there exist $x_{1}, \cdots, x_{k} \in R^{n}$ such that $g_{j}=\hat{x}_{j}$, the linear functional defined by the inner product $\left(x_{j} \mid \cdot\right)$. Let $C$ be the convex hull of $\left\{x_{1}, \ldots, x_{k}\right\}$. $C$ is the intersection of closed half-spaces $H_{a}=\left\{x \in R^{n} \mid\left(x \mid v_{a}\right) \geq b_{a}\right\}$ for some $v_{a} \in R^{n}, b_{a} \in R$. Fix $a$. We have assumed $\bigwedge_{j=1}^{k} g_{j} \leq 0$. Thus $\left(x_{j} \mid v_{a}\right) \leq 0$ for some $j=1, \cdots, k$. Note $x_{j} \in H_{a}$ since $x_{j} \in C$. Thus $b_{a} \leq 0$, and hence $0 \in H_{a}$. We conclude $0 \in C$.

Hence there exist $r_{1}, \cdots, r_{k} \geq 0$ in $R$ with $\Sigma r_{j}=1$ and $\Sigma r_{j} x_{j}=0$. Thus $\Sigma r_{j} \hat{x}_{j}=\left(\Sigma r_{j} x_{j}\right)^{\wedge}$ is the zero functional. Therefore, condition (ii) of Theorem 2.2 is satisfied, and the proof is complete.

Note. The fact that condition (ii) is satisfied in this setting has been of independent interest. An algebraic proof was given already in 1921 by W. Carver (Ann. of Math. 23, pp. 212-220). More recently, it has played a role in the theory of of linear programming.

Henceforth, the vecto: sublattice of $V\left(R^{S}\right)$ generated by the coordinate projections $\pi_{i}, i \in S$, will be denoted by $\operatorname{FVL}(S)$. By Theorem 2.3 it is the free vector lattice with free generating set of cardinality $|S|$. We will ordinarily write FVL $n$ instead of $\operatorname{FVL}(S)^{\prime}$ when $|S|=n$ is finite.

Each $f \in \mathrm{FVL}(S)$ can be written $f=\bigvee_{I} \wedge_{J} f_{i j}$ where $I, J$ are finite and the $f_{i j}$ are linear combinations of the $\pi_{i}, i \in S$. The $f_{i j}$ are linear and continuous since the $\pi_{i}$ are. (We assume the usual product topology on $R^{S}$.) Thus $f$ is continuous and positively bomogeneous $\left(f(r x)=r f(x)\right.$ for all $x \in R^{S}$ and $\left.0 \leq r \in R\right)$. Since $f$ is continuous, $T(f)=\left\{x \in R^{S} \mid f(x) \neq 0\right\}$ is an open subset of $R^{S}$.

Corollary 1. FVL $(S)$ is a vector sublattice of a product of copies of $R$.

Corollary 1 has a number of claimants. An early result along these lines was given by I. Amemiya (J. Fac. Sci. Hokkaido Univ. 12 (1952), 111-156). Corollaries 2-4 are due to E. Weinberg. It seems that Corollary 5 does not, as such, appear in the literature, although it is part of the folklore.

Corollary 2 ([14, Theorem 2] and [1]). No dis joint subset of $\mathrm{FVL}(S)$ is uncountable.

Proof. By definition the subset $Y$ of FVL(S) is disjoint iff $g \wedge b=0$ whenever $g, b \in Y$. Hence $T(g) \cap T(b)=T(g \wedge b)=T(0)=\varnothing$ if $g, b \in Y$. Thus 
$\{T(f) \mid f \in Y\}$ is a pairwise disjoint collection of open subsets of $R^{S}$. Such a collection is necessarily countable. (2) Thus $Y$ is countable. (This proof appeared in [2] and is included here only for completeness.)

Corollary 3 ([14, Theorem 1] and [4, Theorem 3.2]). FVL(S) is indecomposable if $|S| \geq 2$.

Proof. If $0 \neq x \in R^{S}$ then there exists $\pi_{i}, i \in S$, such that $\pi_{i}(x) \neq 0$. Thus $T(\mathrm{FVL}(S))=\bigcup\{T(f) \mid f \in \mathrm{FVL}(S)\}$ is connected if $|S| \geq 2$. Suppose (by way of contradiction) that $\mathrm{FVL}(S)$ is the cardinal sum of nonzero ideals $A$ and $B$. Then $T(A) \cap T(B)=T(A \cap B)=T(0)=\varnothing$, and $T(A) \cup T(B)=T(A \vee B)=T(\mathrm{FVL}(S))$.

Thus $T(\mathrm{FVL}(S))$ is the disjoint union of two nonempty open sets, contradicting connectedness.

Corollary 4 ([13, Theorem 4.2]). FVL(S) bas no basic elements if $|S| \geq 2$.

Proof. By definition $b>0$ is basic iff the set of all $x$ such that $0 \leq x \leq b$ is totally ordered. It is known that in an archimedean vector lattice, the ideal generated by a basic element is a copy of $R$, and, moreover, a cardinal summand [8, Proposition 2.10 and Theorem 3.1]. Hence it follows from Corollary 3 that $\operatorname{FVL}(S)$ has no basic elements if $|S| \geq 2$.

It has long been known that the free vector lattice on one element is the cardinal sum of two copies of $R$. Thus Corollaries 3 and 4 fail if $|S|=1$.

By an open cone in $R^{n}$ we mean an open subset $U$ of $R^{n}$ such that $x \in U$ and $0<r \in R$ imply $r x \in U$.

Corollary 5. Let $X$ be a set, and let $W$ be the vector sublattice of $V(X)$ generated by $g_{1}, \cdots, g_{n}$. If each nonempty open cone in $R^{n}$ bas nonempty intersec. tion with $K=\left\{\left(g_{1}(x), \cdots, g_{n}(x)\right) \mid x \in X\right\}$, then $g_{1}, \cdots, g_{n}$ freely generate $W$.

Proof. Define $\alpha: X \rightarrow K$ by $\alpha(x)=\left(g_{1}(x), \cdots, g_{n}(x)\right)$, and let $\bar{\alpha}:$ FVL $n \rightarrow$ $V(X)$ be given by $\bar{\alpha}(f)=f \circ \alpha$. Then $\bar{\alpha}$ is a vector lattice homomorphism. Suppose $\bar{a}(f)=0$. Then $f \circ a(x)=0$ for all $x \in X$, and hence $f(k)=0$ for all $k \in K$. Thus $f(r k)=r f(k)=0$ for all $k \in K$ and $0 \leq r \in R$. We conclude from the continuity of $f$, and the fact that the set $\{r k \mid k \in K, 0 \leq r \in R\}$ is dense in $R^{n}$, that $f=0$. Thus $\bar{\alpha}$ is one-to-one. Finally, $\bar{\alpha}\left(\pi_{i}\right)(x)=\pi_{i} \circ \alpha(x)=\pi_{i}\left(g_{1}(x), \ldots, g_{n}(x)\right.$. Thus $\bar{\alpha}\left(\pi_{i}\right)=$ $g_{i}$, and the $g_{i}$ freely generate $\operatorname{Im} \bar{\alpha}=W$.

Remark 1. The condition that each nonempty cone in $R^{n}$ intersect $K$ is also necessary. This follows from the arguments in $\$ 4$.

Remark 2. The corollary can be formulated for the infinite generator case

(2) K. Ross and A. Stone, Amer. Math. Monthly 71 (1964), 398-403. 
as follows: Let $W$ be the vector sublattice of $V(X)$ generated by elements $\left\{g_{a} \mid\right.$ $a \in A\}$. If the set $\left\{\left(\operatorname{rg}_{a}(x)\right)_{a \in A} \mid x \in X, 0 \leq r \in R\right\}$ is dense in $R^{A}$, then the $g_{a}$ freely generate $W$.

Remark 3. The question of whether the free vector lattice on continuously many free generators can be represented as real sequences (indexed by the integers) has been of some interest $\left[13, \mathrm{p} .221\right.$, line 1]. It is known that $R^{S}$ contains a countable dense subset iff $|S| \leq c$, the cardinality of the continuum. (3) Hence $\mathrm{FVL}(S)$ can be represented as real sequences whenever $|S| \leq c$.

We turn now to further properties of $\mathrm{FVL}(S)$. There are well-known examples of vector lattices that do not decompose into indecomposable summands.

Theorem 2.4. Each ideal of $\mathrm{FVL}(S)$ is the cardinal sum of indecomposable ideals of $\mathrm{FVL}(S)$.

Proof. Let $A$ be an ideal of $\operatorname{FVL}(S)$, and let $\left\{C_{k}, k \in K\right\}$ be the set of all topological components of $T(A)$. Let $A_{k}=\left\{g \in A \mid T(g) \subseteq C_{k}\right\}$. Then $A_{k}$ is an ideal of $\mathrm{FVL}(S)$.

If $0<g \in A$, then $g$ has an expression $g=\bigvee_{I} \wedge_{I}\left(f_{i j} \vee 0\right)$ where $I$ and $J$ are finite and the $f_{i j}: R^{S} \rightarrow R$ are linear. For each $i \in I$ let $g_{i}=\bigwedge_{J}\left(f_{i j} \vee 0\right)$. Then $0 \leq g_{i} \leq g$, and hence $g_{i} \in A$. Moreover, $T\left(g_{i}\right)=\bigcap_{J} T\left(f_{i j} \vee 0\right)$. Each $T\left(f_{i j} \vee 0\right)$ is convex since $f_{i j}$ is linear, and hence each $T\left(g_{i}\right)$ is convex and, in particular, connected. Hence for each $i \in I$ there is some $k \in K$ such that $T\left(g_{i}\right) \subseteq C_{k}$. Thus $g_{i} \in A_{k}$, and hence $g=\bigvee_{l} g_{i} \in \bigvee_{K} A_{k}$.

Clearly $T\left(A_{k}\right) \subseteq C_{k}$. Let $x \in C_{k}$. Then $x \in T(g)$ for some $0<g \in A$. Write $g=\bigvee_{I} g_{i}$ as in the preceding paragraph. Then $T(g)=\bigcup_{I} T\left(g_{i}\right)$. Hence $x \in$ $T\left(g_{i}\right) \subseteq C_{k}$ for some $i \in I$. Now $g_{i} \in A_{k}$ and $x \in T\left(A_{k}\right)$. Thus $T\left(A_{k}\right)=C_{k}$, and since $C_{k}$ is connected, $A_{k}$ is indecomposable.

Finally, if $k_{1} \neq k_{2}$, then $T\left(A_{k_{1}} \cap A_{k_{2}}\right)=T\left(A_{k_{1}}\right) \cap T\left(A_{k_{2}}\right)=C_{k_{1}} \cap C_{k_{2}}=\varnothing$. Thus the $A_{k}$ are pairwise disjoint, and for vector lattices this is enough for the ir sum to be direct. We have shown that $A$ is the cardinal sum of the indecomposable ideals $A_{k}$.

We seek information about the automorphisms of $\mathrm{FVL}(S)$. We begin with the following two lemmas in which there is a finiteness restriction.

Lemma 2.5. Suppose $a: R^{n} \rightarrow R^{n}$ (n finite) is a vector space isomorphism, and let $f \in \mathrm{FVLn}$. Then $f \circ \alpha^{-1} \in \mathrm{FVLn}$, and $T\left(f \circ \alpha^{-1}\right)=\alpha(T(f))$. The map $a^{*}:$ FVL $n \rightarrow$ FVL $n$ defined by $\alpha^{*}(f)=f \circ \alpha^{-1}$ is a vector lattice automorphis $m$.

Proof. Write $f=\bigvee_{I} \wedge_{J} f_{i j}$ where $I, J$ are finite and the $f_{i j}: R^{n} \rightarrow R$ are linear. Then $f \circ \alpha^{-1}=\bigvee_{I} \wedge_{J}\left(f_{i j} \circ \alpha^{-1}\right)$, a routine verification, and the $f_{i j} \circ a^{-1}$

(3) K. Ross and A. Stone, op. cit. 
are linear. Since $n$ is finite, the $f_{i j} \circ \alpha^{-1}$ are in the subspace of FVLn generated by the coordinate projections. Hence $f \circ a^{-1}$ is in FVLn. The remainder of the proof is quite elementary and is omitted here.

Lemma 2.6. Let $0 \neq f \in \mathrm{FVLn}$. There exist a finite number of automorphisms $\sigma_{1}, \cdots, \sigma_{k}$ of FVL $n$ and a positive integer $m$ such that $\left|\pi_{1}\right| \leq m\left(\left|\sigma_{1} f\right|+\cdots+\left|\sigma_{k} f\right|\right)$.

Proof. Let $0 \neq z \in T(f)$. Suppose $y \in S^{n-1}$, the unit sphere in $\mathbf{R}^{n}$. There exists a vector space isomorphism $\alpha_{y}: \mathbf{R}^{n} \rightarrow \mathbf{R}^{n}$ such that $\alpha_{y}(z)=y$. The sets $T\left(\alpha_{y}^{*} f\right), y \in S^{n-1}$, cover $S^{n-1}$. Hence there exist a finite number of automorphisms $\sigma_{1}, \cdots, \sigma_{k}$ of FVL $n$, where each $\sigma_{i}$ is some $\alpha_{y}^{*}$, such that $s^{n-1} \subseteq$ $T\left(\sigma_{1} f\right) \cup \cdots \cup T\left(\sigma_{k} f\right)=T\left(\left|\sigma_{1} f\right|+\cdots+\left|\sigma_{k} f\right|\right)$. By the compactness of $S^{n-1}$ there is an integer $m$ such that $\left|\pi_{1}\right|(x) \leq m\left(\left|\sigma_{1} f\right|+\cdots+\left|\sigma_{k} f\right|\right)(x)$ for all $x \in S^{n-1}$. Since the elements of FVLn are positively homogeneous, the preceding inequality actually holds for all $x \in R^{n}$.

An ideal of a vector lattice $V$ is characteristic iff it is invariant under all automorphisms of $V$. If the only characteristic ideals of $V$ are the zero ideal and $V$ itself, then $V$ is said to be characteristically simple.

Theorem 2.7. $\mathrm{FVL}(S)$ is characteristically simple.

Proof. Suppose $A$ is a nonzero characteristic ideal of $\operatorname{FVL}(S)$. Since each permutation of the free generators of $\mathrm{FVL}(S)$ induces an automorphism of $\operatorname{FVL}(S)$, if one of the free generators $\pi_{i}$ is in $A$, then they all are, whence $A=\operatorname{FVL}(S)$.

Let $0 \neq f \in A$. Let $\pi_{1}, \cdots, \pi_{n}$ be the finitely many projections occurring in some expression for $f$ in terms of the free generators $\pi_{i}$. The vector sublattice $W$ of $\mathrm{FVL}(S)$ generated by $\pi_{1}, \cdots, \pi_{n}$ is isomorphic to FVL $n_{\text {. By Lemma } 2.6}$ there exist automorphisms $\sigma_{1}, \cdots, \sigma_{k}$ of $W$ such that $\left|\pi_{1}\right| \leq m\left(\left|\sigma_{1} f\right|+\cdots+\left|\sigma_{k} f\right|\right)$. For each $j=1, \ldots, k$ let $\tau_{j}$ be the autormorphism of $\operatorname{FVL}(S)$ such that $\tau_{j}\left(\pi_{i}\right)=\sigma_{j}\left(\pi_{i}\right)$ if $i=1, \cdots, n$, and $\tau_{j}\left(\pi_{i}\right)=\pi_{i}$ otherwise. Since $A$ is characteris tic, each $\tau_{j}(f)$ is in $A$. Thus $m\left(\left|\tau_{1} f\right|+\cdots+\left|\tau_{k} f\right|\right) \in A$. But $\tau_{j}(f)=\sigma_{j}(f)$. Hence $\pi_{1} \in A$. Thus $A=\mathrm{FVL}(S)$, and the theorem is proved.

\section{PART II}

3. Preliminaries. In Part II we shall be concerned exclusively with finitely generated vector lattices. The present section is devoted to reviewing the machinery that will be employed in the subsequent sections.

We recall from $\$ 2$ that the members of FVLn are functions $f: R^{n} \rightarrow R$ where $f=\bigvee_{I} \wedge_{J} f_{i j}$ (pointwise) and the $f_{i j}: R^{n} \rightarrow R$ are linear. The members are, in particular, continuous and positively homogeneous. Since $f$ is positively homogeneous, its values on any ray from the origin in $R^{n}$ are just positive scalar 
multiples of its value at any single point $(\neq 0)$ on the ray. In particular, FVL $n$ is isomorphic to the vector lattice obtained by restricting each element of FVLn to the unit sphere $S^{n-1}$ in $R^{n}$. We will make this identification. FVL $n$ is thus a vector sublattice of the vector lattice $C\left(S^{n-1}\right)$ of all continuous real-valued functions on $S^{n-1}$.

For the remainder of this section, $X$ (with or without subscripts) denotes a compact Hausdorff space. Much of the classical maximal ideal theory of $C(X)$ is retained by vector sublattices of $C(X)$ having enough members. We review a portion of this theory under the standing assumption that $V$ is a vector sublattice of $C(X)$ such that the open sets $T(f), f \in V$, form a base for the topology of $X$ 。

For $x \in X$ we define $\phi_{x}: V \rightarrow R$ by $\phi_{x}(f)=f(x) . \phi_{x}$ is a vector lattice epimorphism, and hence $\operatorname{Ker} \phi_{x}=\{f \in V \mid f(x)=0\}$ is a maximal ideal of $V$. In this way distinct points of $X$ determine distinct maximal ideals of $V$. We will write $M_{x}=\operatorname{Ker} \phi_{x}$. If $A$ is an ideal of $V$ and $T(A)=X$, then $A=V$. Thus the ideals $M_{x}, x \in X$, are the only maximal ideals of $V$ 。

Lemma 3.1. For an ideal $A$ of $V$, the following are equivalent:

(1) $T(f) \subseteq T(A)$ implies $f \in A$ 。

(2) $A$ is an intersection of maximal ideals of $V$ 。

(3) $V / A$ is a subdirect sum of copies of $R$.

(4) $V / A$ is archimedean.

Abbreviated proof. (2) equivalent to (3), and (3) implies (4) are always true for vector lattices. (4) implies (3) since $V$ has a strong unit. Assume (1). Then $A=\bigcap\left\{M_{x} \mid x \in X \backslash T(A)\right\}$. Finally, assume (2). Then $A=\bigcap\left\{M_{x} \mid x \in K\right\}$ for some subset $K$ of $X$. If $T(f) \subseteq T\left(M_{x}\right)$, then $f(x)=0$ and thus $f \in M_{x}$. Hence, if $T(f) \subseteq$ $T(A)$, then $f \in A$ 。

Lemma 3.2. Suppose $A$ is an ideal of $V$ such that $V / A$ is archimedian. Let $K=X \backslash T(A)$. Then $V / A$ is isomorphic to the vector lattice $W=\left\{\left.f\right|_{K} \mid f \in V\right\}$ of restrictions of the members of $V$ to $K$.

Proof. The map restricting $f$ to $\left.f\right|_{K}$ is a vector lattice homomorphism whose kernel, by Lemma 3.1 , is $A$ 。

The reader is referred to [6] for more details and for a proof of the following lemma.

Lemma 3.3. Suppose $V_{1}$ and $V_{2}$ are vector sublattices of $C\left(X_{1}\right)$ and $C\left(X_{2}\right)$, respectively, and suppose $\alpha$ is an isomorphism of $V_{1}$ onto $V_{2^{\circ}}$. Define $\bar{\alpha}\left(x_{1}\right)=x_{2}$ if and only if $\alpha\left(M_{x_{1}}\right)=M_{x_{2}}$. Then $\bar{\alpha}$ is a bomeomorphism of $X_{1}$ onto $X_{2}$ 。

We return to the case of FVLn. The next two lemmas, due to K. Baker, are crucially important. 
Lemma 3.4 [2, Lemma 3.2]. The sets $T(f), f \in \mathrm{FVLn}$, form a base for the topology of $S^{n-1}$.

Lemma 3.5 [2, Lemma 3.3]. If $f, g \in \mathrm{FVLn}$ and $T(f) \subseteq T(g)$, then $|f| \leq m|g|$ for some positive integer $m$. In other words, if $T(f) \subseteq T(g)$ then $f$ is in the principal ideal $\langle g\rangle$ of FVLn generated by $g$.

Remarks. Our statements of the last two lemmas above are somewhat weaker than the original versions. However, they are adequate for our purposes, and avoid the introduction of geometric terminology. It can be said that the arguments in [2] are primarily geometrical in nature, based on certain linearity properties of the elements of FVLn, while the arguments in the present paper are mostly topological.

We conclude these preliminaries with one final lemma. There are, of course, elements $f \in \mathrm{FVL} n$ such that $T(f)=S^{n-1}$ For instance, $f=\left|\pi_{1}\right| \vee \cdots \vee\left|\pi_{n}\right|$. The following is more interesting.

Lemma 3.6. If $x \in S^{n-1}$, then there exists $g \in \mathrm{FVL} n$ such that $T(g)=$ $S^{n-1} \backslash\{x\}$.

Proof. Let $f=\left|\pi_{1}\right| \vee\left|\pi_{2}\right| \vee \cdots \vee\left|\pi_{n-1}\right| \vee-\pi_{n}$. Then $T(f)=S^{n-1} \backslash\{z\}$ where $z=(0, \cdots, 0,1)$. Let $x \in S^{n-1}$, and let $\alpha: R^{n} \rightarrow R^{n}$ be an orthogonal vector space isomorphism such that $a z=x$. Take $g=f \circ \alpha^{-1}$. By Lemma 2.5 we have $g \in \mathrm{FVL} n$, and, moreover, $T(g)=\alpha T(f)=a S^{n-1} \backslash\{\alpha z\}=S^{n-1} \backslash\{x\}$.

4. Maximal ideal theory applied to FVLn $S^{n-1}$ is, of course, a compact connected metric space. A deeper property of spheres is expressed in the following lemma.

Lemma 4.1. Suppose $G$ and $H$ are bomeomorphic subsets of $S^{n-1}$, and $G$ is an open subset of $S^{n-1}$. Then(4)H is an open subset of $S^{n-1}$.

Theorem 4.2. If $A$ is a nonzero ideal of FVLn, then FVLn and FVLn/A are not is omorphic.

Proof. Suppose (by way of contradiction) that FVLn/A is isomorphic to FVLn. By Lemma 3.2 we can consider FVLn/A to be the vector lattice $W$ of restrictions of elements in FVLn to $K=S^{n-1} \backslash T(A)$. The sets $T(f), f \in$ FVLn, form a base for the topology of $S^{n-1}$, and hence the sets $T(g), g \in W$, form a base for $K$. By Lemma 3.3 we conclude $K$ is homeomorphic to $S^{n-1}$. $K$ is a closed subset of $S^{n-1}$, and by Lemma $4.1, K$ is also open. Clearly, $K \neq \varnothing$. Since $S^{n-1}$ is connected, we conclude $K=S^{n-1}$. Thus $T(A)=\varnothing$, contradicting the fact that $A \neq 0$.

(4) J. Hocking and G. Young, Topology, Addison-Wesley, Reading, Mass., 1 961, p. 277. 
Theorem 4.3. Let $T$ be a generating subset of FVLn。T is a set of free generators if and only if $|T|=n$ 。

Proof. Suppose $|T|=n$ 。 Let $\eta:$ FVL $n \rightarrow F V L n$ be an epimorphism carrying the free generators of FVL $n$ onto $T$ 。 Then FVLn/Ker $\eta$ is isomorphic to FVL $n$ 。 We conclude from Theorem 4.2 that $\operatorname{Ker} \eta=0$. Hence $\eta$ is an isomorphism, and thus $T$ is a free set of generators.

The converse has been proved by Weinberg [13, Theorem 2.13].

For example, the elements $\pi_{1}$ and $2 \pi_{2} \vee \pi_{2}$ generate FVL2 since $\pi_{2}=$ $\left(2 \pi_{2} \vee \pi_{2}\right) \wedge 1 / 2\left(2 \pi_{2} \vee \pi_{2}\right)$. Thus they are free generators. Unlike the automorphisms constructed in Lemma 2.5, the automorphism of FVL2 taking $\pi_{1}$ to $\pi_{1}$ and $\pi_{2}$ to $2 \pi_{2} \vee \pi_{2}$ does not leave invariant the subspace of FVL2 generated by $\pi_{1}$ and $\pi_{2}$.

Note. Theorem 4.3 can be used to answer in the finitely generated case the question raised in the first paragraph of p. 222 of [14]. Indeed, if $D$ is a finitely generated distributive lattice, then the "free vector lattice over $D$ " is a free vector lattice if and only if $D$ is free in the category of distributive lattices.

Another application of Theorem 4.3 occurs in the next section.

By Theorem 2.7, FVLn is characteristically simple. Although there exist other finitely generated characteristically simple vector lattices, there is an important class of epimorphic images of FVL $n$ in which this never happens.

If $Y$ is a nonempty subset of a vector lattice $V$, we let $Y^{\prime}=\{x \in V \mid$ $|x| \wedge|y|=0$ for all $y \in Y\}$. $Y^{\prime}$ is an ideal of $V$, called the polar of $Y$ in $V$ 。

Theorem 4.4. If $A=Y^{\prime}$ is a polar in FVLn, and FVLn/A is characteristically simple, then $A=0$ or $A=\mathrm{FVL}$.

Proof. FVL $n / A$ is archimedean since $A$ is a polar and FVL $n$ is archimedean [9, Lemma 3.3]. Let $K=S^{n-1} \backslash T(A)$, and let $V=\left\{\left.f\right|_{k} \mid f \in \mathrm{FVL} n\right\}$. By Lemma $3.2, \mathrm{FVLn} / A$ is isomorphic to $V$, and hence $V$ is characteristically simple. Let $C=\left\{M_{x} \mid x\right.$ is a boundary point of $K$ and $M_{x}$ is the maximal ideal of $V$ associated with $x\}$, and let $J=\bigcap C$. Suppose $\alpha$ is an automorphism of FVLn with inverse $\beta$. Form $\bar{\alpha}$ and $\bar{\beta}$ as in Lemma 3.3, and note $\bar{\alpha}$ and $\bar{\beta}$ are inverses.

Suppose $z$ is an interior point of $K$. Let $U \subseteq K$ be an open subset of $S^{n-1}$ containing $z$. By Lemma $4.1, \bar{\alpha}(U)$ is an open subset of $S^{n-1}$; moreover, $\bar{\alpha}(U) \subseteq$ $\bar{\alpha}(K)=K$. Thus $\bar{\alpha}(z)$ is an interior point of $K$.

Let $f \in J$, and let $x$ be a boundary point of $K . \bar{\beta}(x)$ cannot be an interior point of $K$, for if it were, then by the preceding paragraph $\bar{\alpha}(\bar{\beta}(x))=x$ would be also. Thus $\bar{\beta}(x)$ is a boundary point of $K$. Therefore $M_{\bar{\beta}(x)} \in C$, and $f \in M_{\bar{\beta}(x)}=$ $\beta\left(M_{x}\right)$. Thus af $\in M_{x}$. Hence $J$ is a characteristic ideal of $V$, and so $J=V$ or $J=0$. 
If $J=V$, then $K$ has no boundary points. Hence $K=S^{n-1}$ and then $A=0$; or $K=\varnothing$ and then $T(A)=S^{n-1}$, whence $A=\mathrm{FVL} n$ 。

Now suppose $J=0$. Let $y \in Y$. Then, for all $f \in A$ we have $|y| \wedge|f|=0$, and hence $T(y) \cap T(f)=\varnothing$. Thus $T(y) \cap T(A)=\varnothing$, and therefore $T(y) \subseteq K$. Let $g=\left.y\right|_{K}$. Then $T(g)=T(y)$ is an open subset of $S^{n-1}$ contained in $K$ 。 Thus $T(g)$ contains no boundary points of $K_{\text {. Hence }} g \in M_{x}$ for each $M_{x} \in C_{0}$. Thus $g \in J$, and so $g=0$. Since $T(g)=T(y)$ we conclude $y=0$. Thus $Y=\{0\}$, and hence $A=$ FVLn。

5. An example. Let $E$ be the vector sublattice of $V(R)$ generated by the functions $g(x)=x$ and the constant function 1. The mapping $\tau$ defined by $\tau(g)=$ $g$ and $r(1)=-1$ does not extend to a vector lattice homomorphism of $E$ into $E$ since $1 \geq 0$ and $r(1) \leq 0$. Thus 1 and $g$ are not free generators, and it follows from The orem 4.3 that

(1) $E$ is not is omorphic to FVL2.

It was stated in [1] that $E$ is a copy of FVL2. In correspondence with Professor Amemiya, he has acknowledged this was an error. He pointed out, however, that the vector sublattice of $E$ generated by $a=g \vee(-g-2)$ and $b=$ $(g-1) \vee(-g-1)$ is free with $a$ and $b$ as free generators. Indeed, one easily verifies that the condition in Corollary 5 to Theorem 2.3 is satisfied. We conclude

(2) E contains a copy of FVL2.

Next we prove

(3) $E$ is isomorphic to FVL2 $/\left\langle\pi_{2} \wedge 0\right\rangle$ where $\left\langle\pi_{2} \wedge 0\right\rangle$ is the ideal of FVL2 generated by $\pi_{2} \wedge 0$.

Proof. Let $\sigma:$ FVL2 $\rightarrow E$ be the unique epimorphism such that $\sigma\left(\pi_{1}\right)=g$ and $\sigma\left(\pi_{2}\right)=1$. We note that $\sigma\left(\pi_{2} \wedge 0\right)=\sigma\left(\pi_{2}\right) \wedge 0=1 \wedge 0=0$ and thus $\left\langle\pi_{2} \wedge 0\right\rangle$ $\subseteq$ Ker $\sigma$. Suppose $f \in \operatorname{Ker} \sigma$. We will show $T(f) \subseteq T\left(\pi_{2} \wedge 0\right)$, for then by Lemma 3.5 we conclude $f \in\left\langle\pi_{2} \wedge 0\right\rangle$. Note that $T\left(\pi_{2} \wedge 0\right)$ is the open lower half of the unit circle $S^{1}$. We distinguish three types of open intervals of $S^{1}$, namely,

(i) intervals that are subsets of the first quadrant of the plane,

(ii) intervals that are subsets of the second quadrant of the plane, and

(iii) intervals that are subsets of the upper-half-plane and contain the point $(0,1)$.

Intervals of types (i), (ii), and (iii) are, respectively, the nonzero supports of functions of types

(i) $b=\left(\left(\pi_{1}-r \pi_{2}\right) \vee 0\right) \wedge\left(\left(-\pi_{1}+s \pi_{2}\right) \vee 0\right), 0 \leq r<s \in R$,

(ii) $b=\left(\left(\pi_{1}+r \pi_{2}\right) \vee 0\right) \wedge\left(\left(-\pi_{1}-s \pi_{2}\right) \vee 0\right), 0 \leq s<r \in R$,

(iii) $b=\left(\left(\pi_{1}+r \pi_{2}\right) \vee 0\right) \wedge\left(\left(-\pi_{1}+s \pi_{2}\right) \vee 0\right), 0<s, r \in R$ 。

One readily verifies that for $b$ of each of these types we have $\sigma(b)>0$. 
Suppose $T(f) \nsubseteq T\left(\pi_{2} \wedge 0\right)$. Then $T(f)$ contains some point in the open upper half plane, and hence $T(f)$ contains an interval of type (i), (ii), or (iii); that is, $T(b) \subseteq T(f)$ for some $b$ of type (i), (ii), or (iii). By Lemma $3.5 b$ is in the ideal of FVL2 generated by $f$, and thus $b \in \operatorname{Ker} \sigma$, a contradiction. Thus $T(f) \subseteq$ $T\left(\pi_{2} \wedge 0\right)$ must hold, and hence $f \in\left\langle\pi_{2} \wedge 0\right\rangle$.

Thus $E=\operatorname{Im} \sigma \cong \mathrm{FVL} 2 / \operatorname{Ker} \sigma=\mathrm{FVL} 2 /\left\langle\pi_{2} \wedge 0\right\rangle$.

(4) $E$ is isomorphic to a vector sublattice of FVL2.

Proof. Let $\tau:$ FVL2 $\rightarrow$ FVL2 be the homomorphism such that $\tau\left(\pi_{1}\right)=$ $\pi_{1}$ and $r\left(\pi_{2}\right)=\pi_{2} \vee 0 . \tau\left(\pi_{2} \wedge 0\right)=\tau\left(\pi_{2}\right) \wedge 0=\left(\pi_{2} \vee 0\right) \wedge 0=0$. Thus $\left\langle\pi_{2} \wedge 0\right\rangle \subseteq \operatorname{Ker} \tau$. If $x$ is any point in the top half of the plane, then $\pi_{2}(x)=$ $\left(\pi_{2} \vee 0\right)(x)$; thus $\pi_{1}(x)=\tau\left(\pi_{1}\right)(x)$ and $\pi_{2}(x)=\tau\left(\pi_{2}\right)(x)$, and hence $f(x)=\tau(f)(x)$ for all $f \in$ FVL2. Thus if $f \in \operatorname{Ker} \tau$, then $T(f) \subseteq T\left(\pi_{2} \wedge 0\right)$, and hence $f \epsilon$ $\left\langle\pi_{2} \wedge 0\right\rangle$. Therefore $\left\langle\pi_{2} \wedge 0\right\rangle=\operatorname{Ker} \tau$. Now $E \cong \mathrm{FVL} 2 /\left\langle\pi_{2} \wedge 0\right\rangle \cong \operatorname{Im} \tau$. Thus $E$ is isomorphic to a vector sublattice of FVL2.

Now, combining (1), (2), and (4) we obtain

(5) $E$ is isomorphic to a vector sublattice of FVL2, and FVL2 is isomorphic to a vector sublattice of $E$, but $E$ and FVL2 are not isomorphic.

In particular, we have

(6) FVL2 properly contains a copy of itself.

Finally, we note that the endomorphism $\tau$ in the proof of (4) is, in fact, idempotent since it is idempotent on the generators $\pi_{1}$ and $\pi_{2}$. The image of any idempotent endomorphism of a free vector lattice is a projective vector lattice [12]. We conclude

(7) $E$ is a projective vector lattice.

6. Maximal ideals in finitely generated vector lattices. An ideal $P(\neq V)$ of a vector lattice $V$ is called prime if $x, y \in V$ and $x \wedge y \in P$ imply $x \in P$ or $y \in P$. Numerous equivalent conditions are known [8, Theorem 1.7], among which we mention that the ideals of $V$ containing $P$ form a chain. In particular, every maximal ideal of $V$ is prime.

Theorem 6.1. The maximal ideals of FVLn are precisely those ideals which are both prime and principal.

Proof. Suppose $M$ is a maximal ideal of FVLn. From the discussion in $\$ 3$ we know $M=M_{x}=\{f \in \operatorname{FVL} n \mid f(x)=0\}$ for some point $x \in S^{n-1}$. By Lemma 3.6 there exists $g \in$ FVL $n$ such that $T(g)=S^{n-1} \backslash\{x\}=T\left(M_{x}\right)$. Clearly $g \in M_{x}$. On the other hand, if $f \in M_{x}$ then $T(f) \subseteq T\left(M_{x}\right)=T(g)$, and by Lemma 3.5, $f \in$ $\langle g\rangle$. Thus $M=\langle g\rangle$.

Conversely, suppose $P$ is a principal prime ideal in FVLn. Because $P$ is principal, Lemmas 3.5 and 3.1 imply that $P=\bigcap\left\{M_{x} \mid x \in K\right\}$ for some subset $K$ 
of $S^{n-1}$. Thus $T(P) \subseteq \bigcap_{x \in K} T\left(M_{x}\right)=\bigcap_{x \in K} S^{n-1} \backslash\{x\}$. If $x_{1}$ and $x_{2}$ are distinct points of $S^{n-1} \backslash T(P)$, then by Lemma 3.4 there exist $0 \leq f_{1}, f_{2} \in$ FVLn such that $x_{1} \in T\left(f_{1}\right), x_{2} \in T\left(f_{2}\right)$, and $T\left(f_{1}\right) \cap T\left(f_{2}\right)=\varnothing$; hence $f_{1} \wedge f_{2}=0 \in P$ but neither $f_{1}$ nor $f_{2}$ is in $P$, contradicting the assumption that $P$ is prime. Thus $S^{n-1} \backslash T(P)$ is a singleton, and hence $K$ is also. Thus $P$ is a maximal ideal.

Corollary. If $M$ is a maximal ideal of a finitely generated vector lattice $V$, then $M$ is a principal ideal of $V$.

Proof. Since $V$ is finitely generated, there exists an epimorphism $\nu$ : FVL $n \rightarrow V$ for some $n . \nu^{-1}(M)$ is a maximal ideal of FVL $n$ and hence is principal. Thus $M$ is principal.

Remark. With the aid of [2, Theorem 5.1] Theorem 6.1 can be proved for all finitely generated projective vector lattices.

There was a time when the phenomenon of an archimedean vector lattice admitting a nonarchimedean epimorphic image was thought bizarre. It is now widely known that quite the contrary is true. (Indeed, the free vector lattices are archimedean.) The following result, which can also be obtained from the the ory in [3], is interesting.

Theorem 6.2. Suppose the vector lattice $V$ is finitely generated (as a vector lattice) and archimedean. The following are then equivalent.

(1) Each epimorphic image of $V$ is archimedean.

(2) $V$ bas only a finite number of maximal ideals.

(3) $V$ is isomorphic to the cardinal sum of a finite number of copies of $R$.

Proof. (1) implies (2). If $V$ has an $n$ element generating set, then by Lemma 3.2 we can without loss of generality assume that $V=\left\{\left.f\right|_{K} \mid f \in \mathrm{FVL} n\right\}$ for some closed subset $K$ of $S^{n-1}$. The sets $T(g), g \in V$, form a base for the topology of $K$ since the sets $T(f), f \in \mathrm{FVL} n$, form a base for $S^{n-1}$. Hence there is a one-toone correspondence between the maximal ideals of $V$ and the points of $K$. Our task is to show that $K$ is finite.

If (by way of contradiction) $K$ is infinite, then since $K$ is compact it contains a limit point of itself, say $x . K \backslash\{x\}$ is not compact. Hence there is a collection of sets $T\left(g_{\gamma}\right), \gamma \in C, g_{\gamma} \in V$, which is a covering of $K \backslash\{x\}$ that admits no finite subcover. Let $A=\left\{g \in V \mid\right.$ there exist finitely many $g_{\gamma_{i}}$, say $g_{\gamma_{1}}, \ldots$, $g_{\gamma_{r}}$, such that $\left.T(g) \subseteq \bigcup_{i=1}^{r} T\left(g_{\gamma_{i}}\right)\right\}$. $A$ is an ideal of $V$ and $T(A)=K \backslash\{x\}$.

By hypothesis $V / A$ is archimedean. Hence by Lemma $3.1, A$ is an intersection of maximal ideals of $V$, and since $K \backslash T(A)=\{x\}$, a singleton, we conclude that $A$ is itself maximal. By the corollary to Theorem $6.1, A$ is a principal ideal of $V$, generated by, say, $b \in V$. Hence there exist $g_{\gamma_{1}}, \cdots, g_{\gamma_{r}}$ such that 


$$
T(A)=T(b) \subseteq T\left(g_{\gamma_{1}}\right) \cup \cdots \cup T\left(g_{\gamma_{r}}\right) \subseteq T(A) .
$$

Thus $T(A)=T\left(g_{\gamma_{1}}\right) \cup \cdots \cup T\left(g_{\gamma_{r}}\right)$, contradicting the fact that the covering $\left\{T\left(g_{\gamma}\right), \gamma \in C\right\}$ of $K \backslash\{x\}$ admits no finite subcover.

We conclude that $K$ is finite, and hence that $V$ has only a finite number of maximal ideals.

(2) implies (3). Since $V$ is finitely generated, $V$ has a strong unit. The intersection of the maximal ideals in an archimedean vector lattice with strong unit is always 0 . Let $M_{1}, \cdots, M_{r}$ be the maximal ideals of $V$. Define $\phi$ : $V \rightarrow \Pi V / M_{i}$ by $\phi(x)=\left(x+M_{1}, \cdots, x+M_{r}\right) . \phi$ is a monomorphism since the maximal ideals have intersection 0 . By the Chinese Remainder Theorem (for vector lattices), $\phi$ is also an epimorphism. Each $V / M_{i}$ is a copy of $R$ since $M_{i}$ is maximal in $V$.

(3) implies (1). This is well known.

\section{APPENDIX}

7. Abelian lattice-ordered groups. P. Conrad has proved [7, Corollary II] that the l-subgroup of $\mathrm{FVL}(S)$ generated by the coordinate projections $\pi_{i}$, $i \in S$, is the free abelian l-group FLG(S) on $|S|$ free generators. It is to be expected, then, that the abelian l-group analogues to the vector lattice results in the preceding sections will generally hold. This being the case, it is the exceptions that are more interesting.

There exist finitely generated abelian l-groups possesing maximal ideals that are not principal (although it is the case that principal prime ideals of FLGn are maximal). As a rather simple example, let $G$ be the $l$-subgroup of $V(R)$ generated by the constant function 1 and the function $g(x)=x$. Choose an irrational number $z$. The map $\phi: G \rightarrow R$ by $\phi(f)=f(z)$ is an l-group homomorphism. Ker $\phi$ is hence a maximal ideal of $G$; moreover, $T(\operatorname{Ker} \phi)=R \backslash\{z\}$. There is no element $f \in G$ with $T(f)=R \backslash\{z\}$, and hence $\operatorname{Ker} \phi$ is not a principal ideal of $G$.

The application of Theorem 6.1 in the proof of Theorem 6.2 is crucial. An example is given in [5] of a finitely generated l-group that is not an l-subgroup of any product of a finite number of copies of $R$, but which, nonetheless, admits only archimedean epimorphic images.

The difficulties can be traced to the failure of FLGn to contain the set of all linear functionals $R^{n} \rightarrow R$. Thus the construction of automorphisms in Lemma 1.4 cannot be carried for FLG $n$ without some amendment. As it turns out, it can still be proved that free abelian $l$-groups are characteristically simple. However, Lemma 3.6 fails for FLG $n$. 
For all results excepting those specifically mentioned above; the arguments presented in this paper for vector lattices apply equally well to abelian l-groups.

\section{BIBLIOGRAPHY}

1. I. Amemiya, Countable decomposability of vector lattices, J. Fac. Sci. Hokkaido Univ. Ser. I 19 (1966), 111-113. MR $34 \# 6499$.

2. K. Baker, Free vector lattices, Canad. J. Math. 20 (1968), 58-66. MR 37 \#123.

3. - Topological methods in the algebraic theory of vector lattices, Dissertation, Harvard University, Cambridge, Mass., 1966.

4. S. J. Bernau, Free abelian lattice groups, Math. Ann. 180 (1969), 48-59. MR 39 \#2680.

5. A. Bigard, Contribution à la théorie des groupes réticulé, Dissertation, Université de Paris, 1969.

6. G. Birkhoff, Lattice theory, 3rd ed., Amer. Math. Soc. Colloq. Publ., vol. 25, Amer. Math. Soc., Providence, R. I., 1967. MR 37 \#2638.

7. P. F. Conrad, Free abelian l-groups and vector lattices, Math. Ann. 190 (1971), 306-312. MR $43 \# 7382$.

8. - Lattice ordered groups, Tulane University, New Orleans, La., 1970.

9. - The lateral completion of a lattice-ordered group, Proc. London Math.

Soc. (3) 19 (1969), 444-480. MR 39 \#542.

10. L. Fuchs, Teilweise geordnete algebraische strukturen, Studia MathematicaMathematische Lehrbücher, Band 19, Vandenhoeck \& Ruprecht, Göttingen, 1966.

MR 34 \#4386.

11. M. Henriksen and J. R. Isbell, Lattice-ordered rings and function rings, Pacific J. Math. 12 (1962), 533-565. MR 27 \#3670.

12. D. M. Topping, Some homological pathology in vector lattices, Canad. J. Math. 17 (1965), 411-428. MR $30 \# 4700$.

13. E. C. Weinberg, Free lattice-ordered abelian groups, Math. Ann. 151 (1963), 187199. MR $27 \# 3720$.

14. - Free lattice-ordered abelian groups. II, Math. Ann. 159 (1965), 217-222. MR $31 \# 5895$.

DEPARTMENT OF MATHEMATICS, UNIVERSITY OF KANSAS, LAWRENCE, KANSAS 66044 